\title{
The food frequency intake and eating behaviours of metabolically obese and non obese polycystic ovary syndrome women
}

\author{
Magdalena Czlapka-Matyasik $^{1}$, Aleksadra Bykowska-Derda ${ }^{1}$, Boguslaw Stelcer ${ }^{2}$, \\ Malgorzata Kaluzna ${ }^{3}$, Katarzyna Ziemnicka ${ }^{3}$ and Marek Ruchała ${ }^{3}$ \\ ${ }^{1}$ Institute of Human Nutrition and Dietetics, Poznan University of Life Sciences, Poznan, Poland, \\ ${ }^{2}$ Institute of Human Nutrition and Dietetics, Poznan University of Life Sciences, poznan, Poland and \\ ${ }^{3}$ Department of Endocrinology, Metabolism and Internal Diseases, Poznan University of Medical Sciences, Poznan, \\ Poland
}

\begin{abstract}
Introduction: Normal weight obesity is characterized as regular BMI and excessive body fatness. This type of metabolic obesity may be observed and overlooked in polycystic ovary syndrome women (PCOS), in whom obesity and increased cardiometabolic risk are observed. There are limited data concerning eating habits and lifestyle behaviours of/in normal weight obesity PCOS women. The purpose of this study is to distinguish the food frequency intake and eating habits behaviours of PCOS women characterized by regular weight and body fatness, regular weight and excess fatness and overweight and excess body fatness.
\end{abstract}

Methods: Women with PCOS ( $\mathrm{n}=149$, BMI: $26 \pm 6 \mathrm{~kg} / \mathrm{m}^{2}$, aged $17-44$ years) were divided into three groups according to the BMI and body fatness percentage $(\mathrm{FM} \%)$ : Regular weight and $\mathrm{FM} \%(\mathrm{RW}, \mathrm{n}=64)$, regular weight and high $\mathrm{FM} \%(\mathrm{RWHF}, \mathrm{n}=24)$ and high weight and high FM\% (HWHF, n = 61). FM\% was measured by air displacement plethysmography (BodPod, Life Measurement Inc, Concord, CA). Anthropometrics included weight, height and waist-to-hip-ratio (WHR) were measured. Food frequency intake and lifestyle behaviours were assessed by Dietary Habits and Nutrition Beliefs Questionnaire (KomPAN, The Committee of Human Nutrition, Polish Academy of Science). To assess psychological factors influencing nutrition, My Eating Habits (MEH) Questionnaire was used. To detect the differences between the groups Kruskall-Wallis ANOVA and Mann Whitney-U tests were used.

Results: The results showed that the groups with higher body fatness (HWHF and RWHF) had significantly higher scores of dietary restrictions than the RW group. The RWHF group had significantly lower frequency intake of grains and lower physical activity in relation to other groups. In the group HWHF we indicated the higher risk of eating disorders since scored there higher in emotional overeating section of Eating Habits questionnaire.

Conclusion:The differences in body fatness in PCOS women might by driven by eating behaviours and frequency of intake selected food groups. Individual approach emphasized on dietary coaching of eating and lifestyle habits, might be beneficial to body composition management and cardiometabolic risk reduction in PCOS women.

\section{Conflict of Interest}

There is no conflict of interest 